\title{
然
}

\author{
María Vidales Picazo*
}

\section{ANÁLISIS TEÓRICO DEL ENDEUDAMIENTO EXTERNO}

Según la teoría económica, que un país acceda al endeudamiento externo es positivo porque permite que su consumo y capacidad de inversión no estén limitados a la producción corriente y nacional. Sin embargo, un uso ineficiente de la financiación recibida imposibilitará la generación de recursos suficientes para hacer frente a las obligaciones de pago. En este trabajo se analizan, desde un punto de vista teórico, las crisis de deuda y las crisis financieras y monetarias, dado que son modelos que están plenamente vigentes y su análisis es especialmente relevante para evitar que estos episodios vuelvan a repetirse.

Palabras clave: endeudamiento externo, fuentes de financiación, países en desarrollo y emergentes.

Clasificación JEL: F34, G01, H63.

\section{Introducción}

Los países en desarrollo y emergentes, pese a su gran heterogeneidad, tienen unas características similares: escasez de capital, bajo nivel de ahorro interno y de PIB per cápita, alto crecimiento demográfico, sistema fiscal poco avanzado, mercados financieros poco desarrollados. Esta idiosincrasia es la que les hace fuertemente dependientes de los flujos externos para emprender o consolidar su proceso de desarrollo.

Según Kindleberger (1958) y Nurkse (1995), estas características llevan a las economías menos avanzadas a sufrir el siguiente "círculo vicioso»: presentan niveles muy bajos de inversiones productivas, que imposibilitan el crecimiento económico y favorecen el estancamiento, el cual

\footnotetext{
* Doctora en Economía y Técnico Comercial y Economista del Estado.

Versión de octubre de 2017.
}

reduce las posibilidades de generar beneficios empresariales y mayores salarios, reduciendo la renta disponible e imposibilitando el ahorro interno, lo cual vuelve a desencadenar la escasez de inversiones productivas.

En este contexto, Rosestein-Rodan (1961) acuñó la teoría del «Big Push», por la cual los países en desarrollo necesitan un «gran empuje» que les permita la capitalización de su economía, pudiendo aumentar el gasto en inversión que desemboque en la industrialización del país como medio para superar el subdesarrollo.

Tradicionalmente, la fuente de financiación más utilizada por los países en desarrollo y emergentes ha sido el endeudamiento externo. En el presente artículo se analizan, desde un punto de vista teórico, tanto los beneficios de dicho endeudamiento como sus riesgos y las circunstancias que pueden llegar a generar una crisis de deuda, entendidas estas como la incapacidad de las instituciones públicas o $\triangleright$ 
privadas de un país para hacer frente de forma continuada al servicio de la deuda (Jacobs y Rodriguez-Arana, 2003). Asimismo, también se realizará un repaso teórico de las crisis financieras y monetarias, ya que las mismas están motivadas por el deterioro de los indicadores macroeconómicos y, en la mayoría de los casos, por la falta de fuentes de financiación adecuadas.

\section{Modelo explicativo de las crisis de deuda externa}

\subsection{Análisis teórico de la «optimalidad» del acceso al endeudamiento externo}

El modelo de Obstfeld y Rogoff (1995) demuestra la existencia de una senda intertemporal óptima en función de la evolución futura de la producción.

\section{Supuestos del modelo:}

1. Economía pequeña y abierta, donde el tipo de interés nacional coincide con el tipo de interés internacional.

2. Existe un solo bien que es bien de consumo y de capital. De este modo, el modelo centra la atención en los flujos internacionales agregados de recursos, sin introducir consideraciones de variaciones en los precios relativos de los bienes.

3. El consumidor es representativo y, por tanto, su función de utilidad es aditiva y separable, evitándose así los problemas de agregación de preferencias.

4. El horizonte es infinito, es decir, existe perfecto altruismo intergeneracional.

5. Los agentes tienen previsión perfecta; existe certidumbre acerca del futuro.

\section{Formulación del modelo:}

La función de utilidad tiene como argumentos el consumo en los distintos periodos:

$$
U=U(\mathrm{Co}, \ldots, \mathrm{Ci}, \ldots, \mathrm{Cn})
$$

Donde $\mathrm{Ci}$ es el consumo en el periodo $i \mathrm{y}$, al ser una función aditiva y separable, Ui es independiente de Uj:

$$
U=\sum_{t=0}^{t=\infty} \frac{U C_{t}}{(1+\delta)^{t}}
$$

Donde $\delta$ es la tasa de descuento subjetiva que mide la preferencia por el consumo presente.

Para deducir la restricción presupuestaria se introduce la cuenta corriente $(C A)$, que se representa como la variación en el valor de sus activos netos respecto al resto del mundo, de manera que, si es positiva, el país es acreedor neto del resto del mundo y, si es negativa, el país es deudor neto:

$$
C A_{t}=B_{t+1}-B_{t}=Y_{t}+r B_{t}-C_{t}-G_{t}-I_{t}
$$

Donde $C A_{t}$ es el saldo de la cuenta corriente en $t ; B_{t+1}$ es el valor de los activos netos en el periodo $t+1 ; B_{t}$ es el valor de los activos netos en el periodo $t ; Y_{t}$ es la renta nacional en el periodo $t ; r$ es la tasa de remuneración del capital o tipo de interés; $r B t$ son los rendimientos de los activos en el periodo $t$ o balanza de rentas; $C_{t}$ es el consumo en el periodo $t ; G_{t}$ es el gasto público en el periodo $t ; I_{t}$ es la inversión en el periodo $t$.

A continuación, se obtiene la restricción presupuestaria intertemporal a través de un proceso de sustitución iterativo y aplicando una condición de transversalidad.

$$
\begin{gathered}
\sum_{t=0}^{t=\infty} \frac{1}{(1+r)^{t}}\left(C_{t}+I_{t}\right)=(1+r) B_{0}+ \\
+\sum_{t=0}^{t=\infty}\left(Y_{t}+G_{t}\right) \frac{1}{(1+r)^{t}}
\end{gathered}
$$

1 Por la cual, el valor presente de los activos netos en el infinito es cero. Esta condición implica que en el infinito todas las deudas van a pagarse o a cobrarse, lo cual garantiza un uso óptimo de los recursos. 
Donde $B_{0}$ son los activos exteriores netos acumulados hasta el periodo actual. Si es negativo, significa que el país está endeudado y $B_{0}$ es su stock de deuda.

Para completar el problema de optimización se introduce como restricción presupuestaria la función de producción y la inversión.

$$
Y_{t}=A_{t} F\left(K_{t}\right)
$$

Donde, $A_{t}$ es el nivel tecnológico de la economía en el periodo $t ; K_{t}$ es el capital en el periodo $t ; F\left(K_{t}\right)$ es la función de producción en el periodo $t$.

$$
K_{t+1}=K_{t}+I_{t}
$$

Donde, $K_{t+1}$ es el capital en el periodo $t+1$ (se está asumiendo ausencia de depreciación).

Maximizando [1], sujeto a [2], [3] y [4], se obtienen las condiciones de primer orden:

$$
\text { a) } \frac{U^{\prime}\left(C_{t}\right)}{U^{\prime}\left(C_{t-1}\right)}=\frac{1+\delta}{1+r}
$$

Es la ecuación intertemporal de Euler, que indica las condiciones en las que el consumo presente se cambia por el consumo futuro para conseguir la senda de consumo óptima.

$$
\text { b) } A_{t+1} F^{\prime}\left(K_{t+1}\right)=r
$$

Esta igualdad indica que se invertirá hasta que el stock de capital al final de $t$ tenga una productividad marginal igual al tipo de interés.

La gran implicación de este modelo es que, gracias al recurso al endeudamiento externo, un país en desarrollo puede mejorar su situación a pesar de la falta de recursos internos. Una economía en desarrollo tiene un tipo de interés mayor que el del resto del mundo (porque el capital es relativamente escaso) y, por tanto, la apertura de la economía supondrá la entrada de capitales, que implicará déficits por cuenta corriente en las primeras fases del desarrollo (se endeuda con el exterior), pero, a cambio, suaviza su patrón de consumo y acelera su desarrollo al no tener que limitar su inversión a su ahorro corriente.

En definitiva, el modelo de consumo intertemporal de Obstfeld y Rogoff muestra que el endeudamiento internacional permite aprovechar mejor las posibilidades de inversión sin tener que renunciar al consumo, consiguiendo así una asignación de recursos más eficiente, acelerando el proceso de crecimiento económico (más rápido de lo que el ahorro interno hubiera permitido) y aumentando el bienestar.

\subsection{El problema de la insostenibilidad de la deuda externa}

Ahora bien, el modelo de Obstfeld y Rogoff asume que el endeudamiento obtenido por el país deudor es racional, ya que surge de un proceso de optimización intertemporal en el que las ganancias de bienestar son claras. Sin embargo, este análisis no quiere decir que todo el endeudamiento sea óptimo. A continuación, se expone un modelo dinámico con restricción presupuestaria con el fin de conocer la capacidad de un país a endeudarse. El modelo de Argandoña, Gámez y Mochón (1996) permite afirmar que existe un nivel máximo de volumen de deuda que una economía puede soportar.

Se asume como supuestos del modelo que un Estado no tiene un límite temporal (es decir, su duración es potencialmente infinita) y además dispone de una fuente de ingresos regular como son los impuestos; el límite reside en la relación entre el crecimiento de la economía y el déficit primario (aquél del que se excluye el pago de intereses de deuda). 
El modelo parte de la siguiente restricción presupuestaria, por la que el gasto público solo se puede financiar de tres maneras (recaudación de impuestos, monetización ${ }^{2}$ o emisión de deuda pública):

$$
P_{t} G_{t}+i_{t} B_{t-1}=P_{t} T_{t}+\left(M_{t}-M_{t-1}\right)+\left(B_{t}-B_{t-1}\right)[1]
$$

Donde $P_{t} G_{t}$ es el gasto público en términos nominales; $i_{t} B_{t-1}$ son los pagos de intereses derivados de la deuda total existente al comienzo del periodo $t ; P_{t} T_{t}$ son los impuestos totales en términos nominales; $\left(M_{t}-M_{t-1}\right)$ es la variación de la base monetaria; $\left(B_{t}-B_{t-1}\right)$ es la variación de la cantidad de deuda pública en circulación.

Reorganizando los factores de la ecuación, se puede reescribir la restricción presupuestaria de la siguiente manera:

$$
P_{t} D_{t}+i_{t} B_{t-1}=\left(M_{t}-M_{t-1}\right)+\left(B_{t}-B_{t-1}\right)
$$

Donde $P_{t} D_{t}$ es el déficit primario nominal, es decir, $P_{t} G_{t}-P_{t} T_{t}$

Para conocer el grado de sostenibilidad de la deuda se trabaja con la ecuación en términos del PIB:

$$
\begin{gathered}
d_{t}+i_{t} \frac{B_{t-1}}{P_{t-1} Y_{t-1}}=m_{t}-\frac{M_{t-1}}{P_{t-1} Y_{t-1}} \frac{P_{t-1} Y_{t-1}}{P_{t} Y_{t}}+ \\
+b_{t}-\frac{B_{t-1}}{P_{t-1} Y_{t-1}} \frac{P_{t-1} Y_{t-1}}{P_{t} Y_{t}}
\end{gathered}
$$

Donde $d t=\frac{P_{t} D_{t}}{P_{t} Y_{t}} ; m t=\frac{M_{t}}{P_{t} Y_{t}} ; b t=\frac{B_{t}}{P_{t} Y_{t}}$

Si ahora se define $g$ como la tasa de crecimiento del PIB (que se supone exógena) y $\Pi_{t}$ como la tasa de inflación en el periodo $t$, la ecuación [3] se convierte en:

2 Monetizar consiste en financiar el déficit público mediante la creación de dinero, generalmente a través de compras de deuda pública en el mercado primario por parte de los bancos centrales.

$$
\begin{aligned}
& D_{t}=m_{t}-m_{t-1} \frac{1}{(1+g)\left(1+\Pi_{t}\right)}+ \\
& +b_{t}-\left(1+i_{t}\right) b_{t-1} \frac{1}{(1+g)\left(1+\Pi_{t}\right)}
\end{aligned}
$$

Para simplificar esta ecuación se asumen los siguientes supuestos:

- $m$ es exógena y estable, por lo que se la considera constante.

- Se define la política monetaria como $(1+\theta)=(1+g)\left(1+\Pi_{t}\right)$, es decir, dado un crecimiento de la renta real $(g)$, la política monetaria persigue un determinado objetivo de inflación (П).

- La política fiscal supone una secuencia de déficits primarios constantes: $d_{t}=d$.

- El tipo de interés real $\left(i_{r}\right)$ es exógeno y satisface la ecuación de Fisher, es decir.

$$
1+i_{r t}=\frac{1+i_{t}}{1+\Pi_{t}}
$$

Aplicando estos supuestos a la ecuación [4], se obtiene la siguiente restricción presupuestaria, la cual representa la dinámica del endeudamiento asociada a una política fiscal (d) y a una política monetaria $(\theta)$ :

$$
b_{t}=\left(d-m \frac{\theta}{(1+\theta)}\right)+\frac{1+i_{r t}}{1+g} b_{t-1}
$$

Con esta definición del modelo se puede afirmar que la deuda total en un periodo depende de las decisiones de política fiscal (déficit público que haya que financiar), las decisiones de política monetaria (emisiones de dinero o monetización) y la rémora fiscal de años anteriores, es decir, cómo evoluciona el pago de intereses de la deuda ya emitida. De esta manera, la deuda de un país, tanto nacional como extranjera, será sostenible si el crecimiento de la renta supera al crecimiento de los tipos de interés reales. 


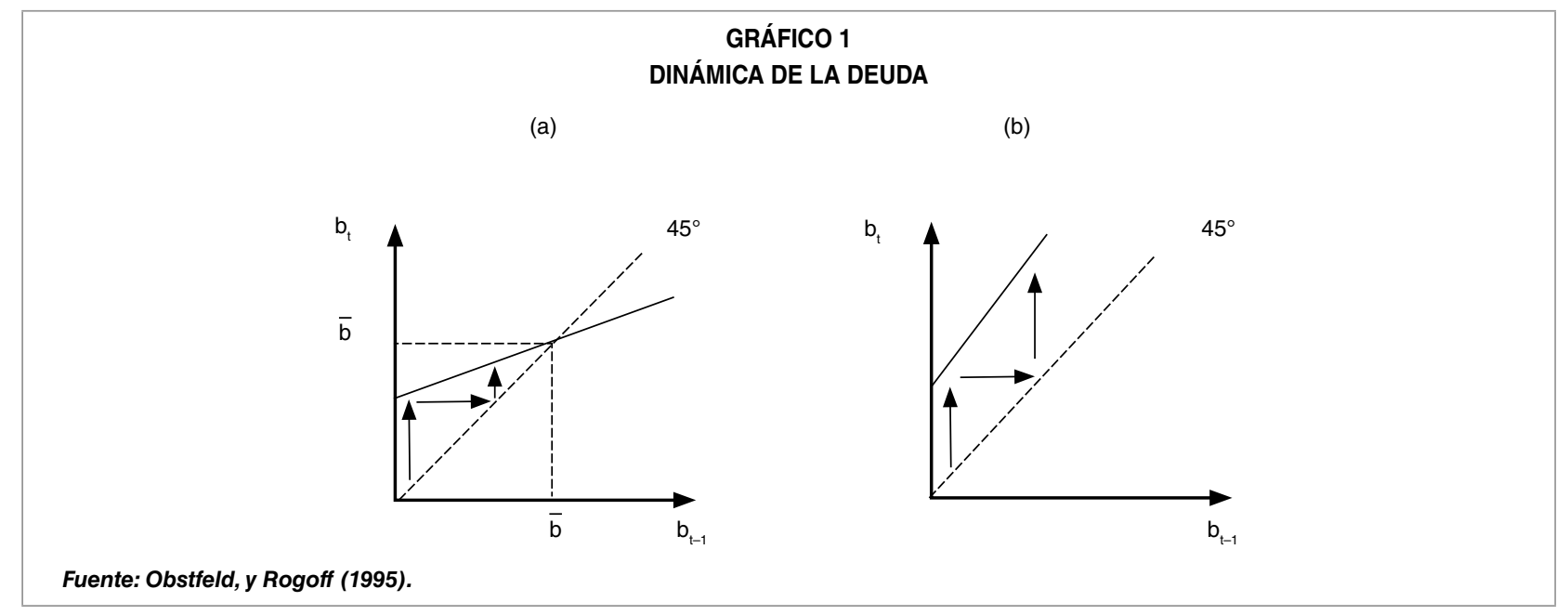

El panel (a) del Gráfico 1 muestra el caso estable en el que el crecimiento del PIB supera al crecimiento de los tipos de interés reales y, por tanto, la deuda converge a un valor estacionario, siendo este valor igual a:

$$
\bar{b}=\frac{\bar{d}-m \frac{\theta}{(1+\theta)}}{1-\frac{1+i_{r t}}{1+g}}
$$

Este estado estacionario (en el cual la ratio deuda-PIB permanece constante a lo largo del tiempo) dependerá de manera positiva del déficit primario $(d)$ y del tipo de interés real $\left(i_{r}\right)$, y de manera negativa, del crecimiento de la renta $(g)$ y de la cantidad de dinero en circulación $(M)$.

El panel (b) representa el caso inestable en el que el crecimiento del PIB es menor que el crecimiento de los tipos de interés reales y la deuda crece de manera explosiva por el mayor coste del pago de intereses, por lo que la trayectoria es divergente y nunca llegará a alcanzar el valor estacionario, generando una situación de insostenibilidad de la deuda.

Muchos países en desarrollo no tienen acceso a los mercados financieros y se ven obligados a monetizar su deuda a través de emisiones de dinero, sin embargo, esta práctica genera fuertes presiones inflacionistas, provocando, en muchos casos, situaciones de hiperinflación. Por ello, cuando un país en desarrollo puede acceder a los recursos que le brindan los mercados financieros internacionales, hace uso de ellos a través de la emisión de deuda, que, en un número muy elevado de casos, es adquirida por inversores internacionales, ya que los niveles de ahorro interno de estos países son muy bajos. Sin embargo, tal y como demuestra el modelo de Argandoña et al. (1996), si los recursos recibidos con dicha financiación exterior no se utilizan para generar inversiones productivas que permitan que la tasa de crecimiento del PIB supere la tasa a la que crece la deuda, la dinámica de esta se volverá insostenible en el largo plazo.

En conclusión, tan importante es conocer las ventajas del endeudamiento como sus riesgos, ya que el acceso a la financiación (tanto nacional como extranjera) puede llevar a una mayor laxitud en las políticas macroeconómicas que terminen desembocando en problemas de insostenibilidad de deuda. En efecto, para evitar situaciones de crisis de deuda, los países endeudados deberán hacer esfuerzos por: fomentar la consolidación fiscal a través de la reducción paulatina del déficit $\triangleright$ 
primario; aumentar la capacidad recaudatoria de los impuestos a través de la lucha contra el fraude fiscal; implementar políticas destinadas al gasto productivo (investigación y desarrollo, capital humano, infraestructuras, etcétera), desarrollar un sistema fiscal no desincentivador de la inversión, evitar el riesgo moral en el sistema financiero y desarrollar un amplio marco regulatorio y de supervisión de los mercados financieros.

\section{Modelos tradicionales de crisis financieras y monetarias}

Una vez analizado cómo pueden originarse las crisis de deuda, a continuación, se analizan los modelos tradicionales de primera, segunda y tercera generación, los cuales tienen como común denominador la importancia del endeudamiento con el exterior, al ser uno de los fundamentos macroeconómicos que al deteriorarse desencadena la crisis.

\subsection{Modelos de primera generación}

Los modelos de primera generación explican las crisis de balanza de pagos por el deterioro de las variables fundamentales (saldo presupuestario, niveles de deuda externa, balanza de pagos, inflación, desempleo...), debido principalmente a la puesta en práctica de políticas demasiado laxas y expansivas que llevan a la imposibilidad de mantener la paridad cambiaria y al lanzamiento de ataques especulativos por parte de los inversores para beneficiarse del colapso anticipado de la moneda (Rodríguez Prada, 2003).

A continuación, se expone el modelo de Flood y Garber (1984), que parte de los siguientes supuestos:
1. El régimen cambiario es de tipos de cambio fijo y el déficit público es exógeno. El banco central está obligado a monetizar el déficit para mantener el tipo de cambio.

2. Los agentes son racionales con previsión perfecta.

3. La economía es pequeña y abierta. Los precios extranjeros $\left(P^{\star}\right)$ y los tipos de interés extranjeros $\left(i^{\star}\right)$ son constantes (por ello aparecerán sin subíndices temporales).

4. Se cumple la paridad del poder adquisitivo (PPA) por la que el tipo de cambio (s) entre las monedas de dos países es igual a la relación entre los niveles de precios $\left(P\right.$ y $\left.P^{\star}\right)$ de esos dos países. Es decir, una disminución del poder adquisitivo de la moneda nacional estará asociada a una depreciación equivalente de su moneda en el mercado de divisas.

$$
P_{t}=s P^{*}
$$

5. Se cumple la paridad no cubierta de intereses, por la que el tipo de cambio entre la moneda nacional y la extranjera (tasa de variación esperada del tipo de cambio) ajustará el beneficio de forma que el tipo de interés en moneda nacional de los depósitos nacionales (i) será igual al tipo de interés en moneda nacional de los depósitos extranjeros $\left(i^{\star}\right)$.

$$
\frac{\dot{s}_{t}}{s_{t}}=i-i^{\star}
$$

6. La base monetaria $(M)$ está compuesta por el valor en moneda nacional de las reservas de divisas $(R)$ y el crédito interno $(C l)$. Asimismo, el crédito interno crece a una tasa $\theta$, que se considera positiva.

$$
\begin{gathered}
M=R+C l \\
\dot{C} l=\theta \quad \theta>0
\end{gathered}
$$


La demanda de saldos reales de dinero depende del tipo de interés de manera inversa:

$$
\frac{M_{t}}{P_{t}}=\alpha-\beta i \quad \alpha, \beta>0
$$

Sustituyendo en la función de demanda de dinero [5], la paridad del poder adquisitivo [1] y la paridad no cubierta de intereses [2] y asumiendo, por simplicidad, que $P^{\star}$ e $i^{\star}$ son iguales a 1, se obtiene la siguiente ecuación del tipo de cambio:

$$
s_{t}=\frac{M_{t}}{\mu}+\frac{\Omega}{\mu} \dot{s}_{t}
$$

Donde $\mu=\Omega P^{*}-\beta P^{*} i^{*}>0 ; \Omega=\beta P^{*}$

Dado que el tipo de cambio es fijo, la variación de este $\left(s_{t}\right)$ es cero y se define de la siguiente manera:

$$
\bar{s}=\frac{M_{t}}{\mu}
$$

Donde $\bar{s}$ es el tipo de cambio fijo, de manera que $M$ es constante y, dada la composición de la base monetaria [3], $\mathrm{Cl}$ aumentará en la misma cuantía en la que disminuye $R$ y viceversa.

En un contexto de tipos de cambio fijos con un déficit público exógeno, los agentes (racionales y con previsión perfecta) saben que se producirá una reducción paulatina de las reservas para mantener el tipo de cambio, y en el momento en que se produzca su agotamiento el tipo de cambio fijo se abandonará y pasará a ser flexible.

A continuación, se determina gráficamente el momento en el que el tipo de cambio se agota, el cual se alcanza en dos etapas (Gráficos 2 y 3).

El Gráfico 2 muestra la evolución del tipo de cambio vigente $(\bar{S})$ y el tipo de cambio sombra (S) (el tipo de cambio que existiría si la moneda flotara de forma natural, sin intervención para

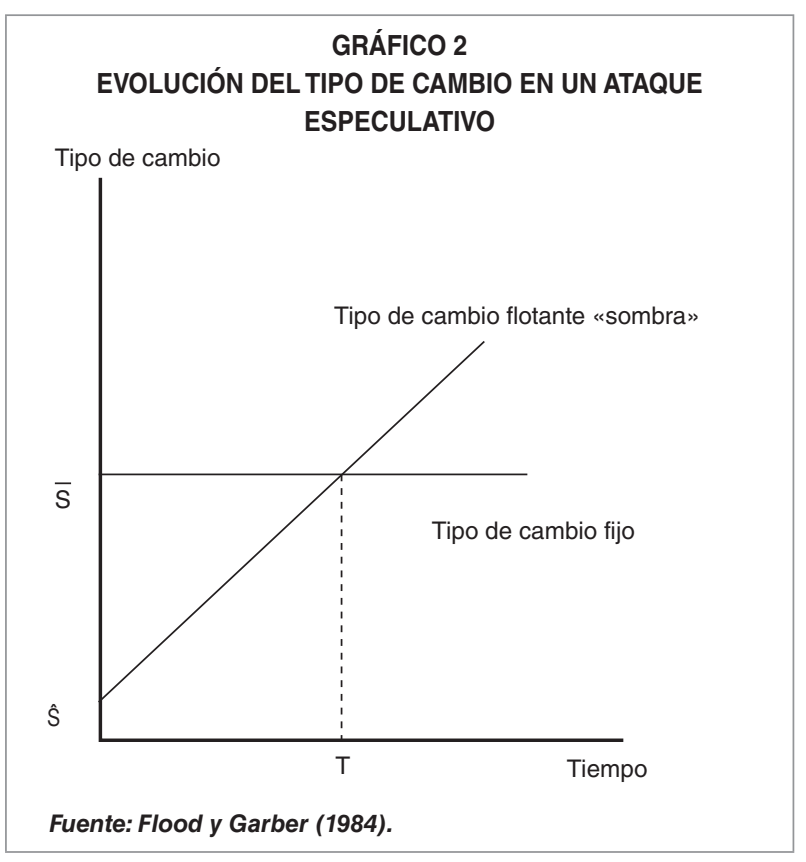

GRÁFICO 3

EVOLUCIÓN DE LA CANTIDAD DE DINERO, EL CRÉDITO INTERNOY LAS RESERVAS EN UN ATAQUE ESPECULATIVO

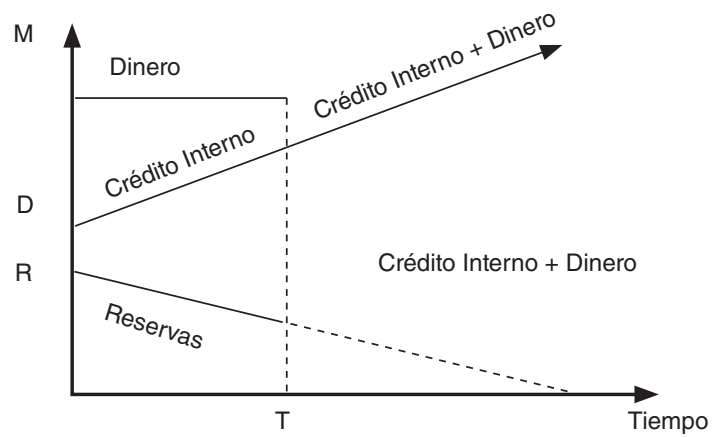

Fuente: Flood y Garber (1984).

defender el tipo de cambio fijo). Antes del ataque, el tipo de cambio es fijo y sin expectativas de depreciación, pero en el momento en que se agotan las reservas $(T)$ el tipo de cambio vigente pasa a ser $\hat{S}$. El momento $T$ es una variable endógena que depende positivamente de la cantidad de reservas de divisas que posea la autoridad monetaria y, negativamente, de la tasa de crecimiento del crédito interno y las elasticidades de la demanda de dinero con respecto al tipo de interés (Rodriguez Prada, 2003). 
El Gráfico 3 muestra la evolución de las principales variables (cantidad de dinero, crédito interno y reservas), antes y después del ataque especulativo. Antes del ataque, la cantidad de dinero es constante (solo varía su composición: mayor crédito interno y menores reservas, en la misma proporción). Sin embargo, dada la situación de las variables fundamentales, los agentes racionales saben que llegará el momento de colapso natural de la economía $(T)$ y actúan antes para obtener ganancias. De tal manera que, en el momento del ataque, las reservas se agotan y la cantidad de dinero se iguala al crédito interno, creciendo, a partir de ese momento, a su mismo ritmo.

La conclusión a la que llega este modelo de primera generación es que las crisis no son un suceso aleatorio, sino que dependen de la consistencia y fortaleza de las variables fundamentales. De tal manera que los ataques especulativos son racionales y predecibles porque los agentes buscan obtener el máximo beneficio a través del arbitraje, generando una crisis cambiaria al ser insostenible el tipo de cambio fijo.

Los modelos de primera generación dan explicación a las crisis cambiarias, las cuales no son consecuencia de ataques de pánico irracionales. Estos modelos sirvieron para explicar la crisis de México (1982), la crisis de Rusia (1998) y la de Argentina (2001).

Sin embargo, estos modelos tienen ciertas limitaciones. Por un lado, algunos de sus supuestos (agentes racionales con previsión perfecta y una autoridad que no se preocupa de la sostenibilidad del tipo de cambio a largo plazo) son demasiado restrictivos. Por otro lado, estos modelos no sirven para explicar las sucesivas crisis de la década de los noventa (Sistema Monetario Europeo en 1992 y Sudeste Asiático en 1997). Estos inconvenientes dieron lugar al surgimiento de nuevas teorías explicativas de las crisis financieras, como los modelos de segunda y tercera generación, en los que la disciplina fiscal y monetaria y unos sólidos fundamentos macroeconómicos no siempre constituyen una garantía suficiente para evitar una crisis financiera (Barberá y Blanca, 2004).

\subsection{Modelos de segunda generación}

En estos modelos, la crisis no es consecuencia del deterioro continuo de los fundamentos que lleva a un agotamiento de las reservas, sino que se desencadena por la decisión del Gobierno de abandonar la paridad del tipo de cambio. Esto se debe a que el coste (en términos de inflación, desempleo y demás variables macroeconómicas) de mantener la paridad y resistir a la presión especulativa es superior a la pérdida de reputación de abandonar el tipo de cambio. En estos modelos el Gobierno tiene un papel activo y, por tanto, su comportamiento no es lineal (al contrario de lo que ocurre en los modelos de primera generación, donde la política gubernamental es pasiva y no se adapta a las expectativas de los agentes), dando lugar a equilibrios múltiples, ya que existe una función de reacción que incorpora la respuesta del Gobierno ante las actuaciones de los agentes y la economía se situará en un equilibrio $u$ otro en función de las expectativas de dichos agentes.

A continuación, se expone el modelo de Obstfeld (1996) de expectativas que se autocumplen. Los supuestos de partida son:

1. Economía pequeña y abierta.

2. Se cumple la paridad del poder adquisitivo (explicada en el apartado anterior).

3. Los precios extranjeros son iguales a 1 (normalizados), de manera que la $D$ 
variación de los precios coincidirá con la variación del tipo de cambio. Por tanto, en un contexto de tipos de cambio fijos, la inflación será nula.

$$
P=s P^{*} \quad P=\dot{s} \Pi=s
$$

Donde $P$ son los precios nacionales; $P^{\star}$ son los precios extranjeros; $s$ es el tipo de cambio, $\Pi$ es la inflación; $\dot{s}$ es la variación del tipo de cambio.

Se parte del modelo básico de Kydland y Prescott (1977) de inconsistencia dinámica de la política monetaria, al que se le añade una «cláusula de escape» por la cual el Gobierno tiene la posibilidad de incumplir su compromiso de defensa de la paridad cambiaria (y, por tanto, devaluar) a cambio de minimizar las pérdidas sociales.

En este contexto, el Gobierno minimiza una función de pérdidas sociales (que, como se ha indicado anteriormente, no es una función lineal, sino cuadrática):

$$
L^{S}=\left(g-g^{\star}\right)^{2}+\beta\left(\Pi-\Pi^{\star}\right)^{2}+C(\Pi)
$$

Donde:

$L^{S}$ es la función de pérdidas sociales del Gobierno (función de reacción).

$g-g^{*}$ es la desviación del crecimiento respecto a los objetivos del Gobierno.

$\beta$ es un coeficiente que representa la aversión del Gobierno a la inflación.

$\Pi-\Pi^{*}$ es la desviación de la inflación respecto a los objetivos del Gobierno.

$C(\Pi)$ es el coste reputacional o coste de realineamiento y representa por tanto la «cláusula de escape». El Gobierno incurre en una serie de costes en términos de reputación y pérdida de credibilidad al desviarse de sus compromisos cambiarios, tolerando una mayor inflación. Este coste reputacional se divide en dos: $C_{D}$, que es el coste de devaluar y $C_{R}$, que es el coste de revaluar.
El output gap de la economía será positivo y vendrá definido por:

$$
k=g^{*}-g_{N}
$$

Donde $g_{N}$ es el crecimiento natural de la economía.

El funcionamiento de la economía vendrá determinado por la curva de Lucas:

$$
g=g_{N}+a\left(\Pi-\Pi^{e}\right)+\varepsilon
$$

Donde $\Pi^{e}$ es la inflación esperada; a es un coeficiente que mide la sensibilidad del crecimiento de la economía ante la desviación de la inflación respecto a la esperada; $\varepsilon$ es la volatilidad de la economía.

Esta función supone que desviaciones de la renta respecto a la renta natural se deben a depreciaciones inesperadas y a shocks de oferta aleatorios (representados por $\varepsilon$ ).

Una vez definidos los supuestos y las ecuaciones del modelo, se puede deducir el problema de optimización al que se enfrenta el Gobierno:

$\operatorname{Min}[2] L^{S}=\left(g-g^{\star}\right)^{2}+\beta\left(\Pi-\Pi^{*}\right)^{2}+C(\Pi)$

s.a. [3] $k=g^{*}-g_{N}>0$

$$
\text { [4] } g=g_{N}+a\left(\Pi-\Pi^{e}\right)+\varepsilon
$$

Para resolver el problema se parte de dos escenarios, que acontecen por la existencia de agentes especuladores:

\section{Shock de oferta negativo muy grave $\left(\varepsilon^{-}\right)$}

Si $\varepsilon^{-}<\varepsilon_{D}$ entonces: $L^{S}{ }_{\text {tc Fijo }}>L_{\text {tc Flexible }}+C_{D} \mathrm{y}$ por tanto $\uparrow s$

Es decir, si el shock es más negativo que el derivado de una devaluación, las pérdidas sociales de mantener el tipo de cambio fijo superan a las pérdidas de pasar al tipo de $\square$ 
cambio flexible más los costes reputacionales y, por tanto, el Gobierno decide devaluar (mantener la paridad tendría un coste social mayor).

\section{Shock de oferta positivo muy favorable $\left(\varepsilon^{+}\right)$}

Si $\varepsilon^{+}>\varepsilon_{R}$ entonces: $L^{S}{ }_{t c \text { Fijo }}>L_{\text {tc Flexible }}^{S}+C_{R} \mathrm{y}$ por tanto $\downarrow S$

Al contrario del escenario anterior, si el shock es tan positivo que supera a los efectos de una revaluación, las pérdidas sociales de mantener el tipo de cambio fijo superan a las pérdidas de pasar al tipo de cambio flexible más los costes reputacionales y, por tanto, el Gobierno decide revaluar.

Solo si $\varepsilon_{R}>\varepsilon^{-/+}>\varepsilon_{D}$, entonces el Gobierno defenderá el tipo de cambio fijo frente al shock, es decir, existe un intervalo de $\varepsilon$ en el que el Gobierno está dispuesto a mantener el tipo de cambio fijo.

El motivo por el que se pueden alcanzar equilibrios múltiples es que los agentes reformulan sus expectativas de inflación. La inflación esperada será el sumatorio de la inflación en caso de revaluación, ponderada por la probabilidad de un shock positivo de mayor valor absoluto que $\varepsilon_{R}$, y la inflación en caso de devaluación, ponderada por la probabilidad de que se devalúe (si el shock negativo es mayor que $\varepsilon_{D}$ ).

$$
\begin{aligned}
\Pi^{e} & =E\left[\Pi \mid \varepsilon^{+}>\varepsilon_{R}\right] \operatorname{Pr}\left(\varepsilon^{+}>\varepsilon_{R}\right)+ \\
& +E\left[\Pi \mid \varepsilon^{-}<\varepsilon_{D}\right] \operatorname{Pr}\left(\varepsilon^{-}<\varepsilon_{R}\right)
\end{aligned}
$$

Si los agentes dan probabilidades altas a que la inflación esperada crezca mucho (lo suficiente como para que llegue a superar la inflación en $t$ ), el crecimiento de la economía en $t$ será menor al crecimiento natural y, para evitar los costes sociales que esto generaría en términos de inflación, desempleo, pérdida de competitividad, etcétera, el Gobierno decide devaluar, lo que implica una mayor inflación, cumpliéndose así las expectativas de los agentes (self-fulfilling expectation o expectativas que se autocumplen).

$$
\Delta \Delta \Pi^{e} \rightarrow \Pi^{e}>\Pi_{t} \rightarrow g_{t}<g_{N} \rightarrow \Delta \Delta s \rightarrow \Pi_{t}
$$

El modelo de Jeanne y Bensaid (1997) establece que la probabilidad de la devaluación depende de los factores de forma no lineal y por ello se generan equilibrios múltiples. De esta manera, pueden coexistir dos equilibrios posibles: un «equilibrio sin ataque» (donde los agentes no se han puesto de acuerdo para coordinar sus políticas a pesar de que existan potenciales ganancias de capital si el ataque se produjera) y «un equilibrio de crisis» (en caso de que los agentes compartan información y coordinen sus políticas, o bien exista un agente con suficiente poder de mercado $^{3}$ ). La conclusión de los modelos de segunda generación es que los fundamentos no generan la crisis, pero sí contribuyen a hacerla más o menos probable, por lo tanto, no se puede establecer una absoluta dicotomía entre las crisis de fundamentos y las crisis de expectativas que se autocumplen. De esta manera, en los modelos de primera generación, los mercados anticipan la crisis, mientras que, en los modelos de segunda generación, son los mercados los que la provocan, si bien los Gobiernos son los que deciden cuándo va a ocurrir (Bajo, 2001). Esto es lo que sucedió en la crisis del Sistema Monetario Europeo (SME) en 1992 (Vidales, 2015). El inconveniente de los modelos de segunda generación es que no explican las crisis acontecidas en el Sudeste Asiático y otras economías emergentes a finales del siglo $\mathrm{xx}$ (Barberá y Clara, 2004); es por ello que surgen los modelos de tercera generación.

3 Como ocurrió con el ataque llevado a cabo por el inversor de origen húngaro George Soros contra la libra esterlina el 16 de septiembre de 1992 (acontecimiento conocido como el «miércoles negro»). 


\subsection{Modelos de tercera generación}

Los modelos de tercera generación reflejan muy bien la relación entre las crisis bancarias y las crisis cambiarias (de ahí que a estos modelos también se les conozca como «modelos de crisis gemelas»). La causalidad entre ambas puede ir en cualquier dirección, aunque la más habitual tiene su origen en la crisis bancaria, y generalmente después de la liberalización financiera (Kaminsky y Reinhart, 1999).

Cuando el sector financiero se encuentra con problemas para cubrir sus obligaciones frente a sus inversores, el banco central actúa de prestamista de última instancia y acude en su ayuda (generalmente a través de la emisión de dinero). Esto genera el incumplimiento del compromiso de tipo de cambio y, por tanto, la crisis cambiaria. Kaminsky y Reinhart (1999) consideran que el origen de las crisis bancarias acontece cuando se produce el cierre, fusión o la compra por parte del sector público de una o más entidades financieras, generalmente porque se ha producido una importante fuga de capitales (como ocurrió en América Latina y el Sudeste Asiático en los noventa o ha ocurrido en Europa con la crisis financiera de 2009).

En el otro sentido, si acontece un shock externo, por ejemplo, una subida de tipos de interés en el exterior, se produce una disminución de las reservas para defender la paridad. Si esta disminución no viene acompañada de una esterilización ${ }^{4}$, la base monetaria y el crédito interno se contraerán, generando una situación de falta de liquidez que desencadenará la bancarrota. Estas crisis gemelas pueden deberse a tres causas principales: el riesgo

\footnotetext{
4 La esterilización monetaria es una medida de política económica utilizada por los bancos centrales para evitar que la entrada o salida de divisas de un país por la propia evolución del mercado altere la masa monetaria (cantidad de dinero en circulación) de dicho país.
}

moral, la fragilidad financiera y el «efecto hoja de balance».

Paul Krugman (1999) explica en profundidad el «efecto hoja de balance» en el modelo que se desarrolla a continuación. El modelo parte de los siguientes supuestos:

- Economía abierta y pequeña, donde las exportaciones se determinan exógenamente.

- Producción de un único bien homogéneo $(Y)$ haciendo uso de dos factores: capital $(K)$ y trabajo $(L)$. Función de producción Cobb-Douglas: $Y_{t}=K_{t a} L_{t 1-a^{*}}$

- El capital dura un solo periodo, de tal manera que el capital en $t$ será igual a la inversión llevada a cabo en el periodo $t-1$.

- El bien $Y$ no es un sustitutivo perfecto de los bienes extranjeros. La elasticidad de sustitución entre los bienes nacionales y los extranjeros es igual a 1 , destinando una fracción $\mu$ a consumo e inversión de bienes extranjeros (importaciones) y la fracción restante $(1-\mu)$ a bienes domésticos.

- Existen dos tipos de residentes: los trabajadores que consumen todo su ingreso $y$, por tanto, tienen un papel pasivo en el proceso de inversión; y los empresarios, que son los que crean e invierten el capital.

- El valor de las exportaciones en términos de bienes extranjeros $(X)$ está exógenamente dado y equivale a $P X$ en términos de bienes nacionales. Siendo $P$ el tipo de cambio real.

Con los supuestos anteriores, en el equilibrio, la producción (oferta) del bien $Y$ es igual a su demanda: 


$$
Y_{t}=(1-\mu) C_{t}+(1-\mu) I_{t}+P_{t} X
$$

Donde $C_{t}$ es el consumo en el periodo $t$ y $l_{t}$ es la inversión en el periodo $t$.

La ecuación [1] se puede reescribir de la siguiente manera, teniendo en cuenta que los trabajadores gastan todo su ingreso $\left[(1-a) Y_{t}\right]$ :

$$
Y_{t}=(1-\mu) I_{t}+(1-\mu)(1-\alpha) Y_{t}+P_{t} X
$$

De la ecuación [2] se puede obtener la expresión que modeliza el tipo de cambio real:

$$
P_{t}=\frac{[1-(1-\mu)(1-\alpha)] Y_{t}-(1-\mu) I_{t}}{X}
$$

Para determinar el nivel de inversión, el modelo parte de que este estará limitado por la capacidad de endeudamiento, o, dicho de otra manera, por el nivel máximo que los acreedores estén dispuestos a prestar. Si se considera que los empresarios pueden pedir prestado como máximo $\theta$ veces su riqueza, la ecuación que modeliza el nivel de inversión financiable sería:

$$
I_{t f}=(1+\theta) W_{t}
$$

Donde $W_{t}$ es la riqueza del empresario y viene expresada como la diferencia entre todo el capital de la economía y las deudas con los prestamistas tanto nacionales $(D)$ como extranjeros $(F)$ :

$$
W_{t}=a Y_{t}-D_{t}-P_{t} F_{t}
$$

Por último, las decisiones de inversión se toman en función de la relación entre la rentabilidad de la inversión doméstica (i) y la rentabilidad de un proyecto de inversión en el extranjero $\left(i^{\star}\right)$. La ecuación [6] refleja que, para que haya inversiones domésticas, la tasa de retorno de estas debe ser al menos tan alta como la tasa de retorno de la inversión exterior:

$$
(1+i) \frac{P_{t}}{P_{t+1}} \geq 1+i^{*}
$$

De todo lo anterior, y teniendo en cuenta que el tipo de cambio real depende de la inversión [3], se puede deducir que existe una relación entre la inversión y la riqueza. De tal manera que la inversión financiada con préstamo [4] permite conocer cómo varía esta cuando varía la inversión (se prescinde de los subíndices por simplicidad):

$$
\frac{d l_{f}}{d l}=\frac{d l_{f}}{d w} \frac{d w}{d l}=\frac{(1-\theta)(1-\mu) F}{X}
$$

Esta ecuación permite explicar cuándo se producirá la crisis. Si la derivada es inferior a la unidad, una economía productiva no experimentará una crisis, incluso aunque tenga problemas de ajuste del stock de capital derivado de las restricciones financieras. Sin embargo, si el resultado de la derivada es mayor que la unidad, puede haber equilibrios múltiples que desemboquen en una crisis, ya que una pérdida de confianza por parte de los prestamistas podrá originar una menor entrada de capitales, causando una caída en el tipo de cambio y afectando al balance (fuertemente apalancado) de los empresarios, llevándoles a la bancarrota.

Los determinantes de la crisis son, por tanto, un alto nivel de apalancamiento $(\theta)$, una elevada ratio de deuda en moneda extranjera respecto a las exportaciones $(F / X)$ y una reducida propensión marginal a importar $(\mu)$. Cada una de estas variables aumenta la posibilidad de que la ecuación [7] sea mayor que la unidad. Este modelo explica en gran medida por qué aconteció la crisis asiática (las economías afectadas por la crisis tenían unos niveles muy elevados de apalancamiento - sobre todo a corto plazo - y muchos pasivos en moneda extranjera).

Una peculiaridad de este modelo, la cual puede llegar a explicar la crisis asiática, es $\triangleright$ 
que se pueden alcanzar puntos de equilibrio localmente estables, pero globalmente inestables, de tal manera que una perturbación lo suficientemente grande de la inversión esperada (provocada, por ejemplo, por un cambio súbito en las expectativas) puede generar una ola de pesimismo que suponga un movimiento drástico desde un punto de equilibrio a otro. Esta evolución de la economía no viene determinada por malas decisiones de inversión o inversiones insolventes, sino por la fragilidad financiera y los «comportamientos de rebaño» (Gandolfo, 2001).

En conclusión, las crisis gemelas pueden ser resultado de diversos factores: movimientos internacionales de capital, liberalización financiera prematura (sin haber garantizado antes unos mecanismos de supervisión y regulación apropiados), problemas de liquidez a nivel internacional, shocks macroeconómicos o la rigidez de los tipos de cambio para conseguir anclar la inflación (Alonso y Blanco, 2004). Kaminsky y Reinhart (1999) concluyen que existen fuertes evidencias de círculos viciosos, donde el colapso de la moneda muy a menudo termina dañando un frágil sistema bancario. En ambos tipos de crisis, un shock financiero (probablemente causado por la liberalización financiera o el acceso cada vez mayor a los mercados de capitales) termina creando una burbuja al proporcionar fácil acceso a la financiación. Estos autores también constataron que cuando ambas crisis terminan aconteciendo, los efectos finales son mucho más severos y recesivos que si ocurren por separado.

\subsection{Inestabilidad financiera de Minsky}

Finalmente, otra aportación teórica muy relevante a la hora de entender las crisis financieras es la hipótesis de inestabilidad financiera de Minsky (1993), que analiza el impacto de la deuda en el comportamiento del sistema económico ${ }^{5}$ y define la existencia de ciclos endógenos producidos por periodos de prolongada prosperidad económica. En momentos de auge económico, cualquier cambio positivo en las expectativas aumenta la demanda de bienes de capital y, consecuentemente, la demanda de créditos. Para hacer frente a esa mayor demanda, los bancos crean nuevos productos financieros (la llamada innovación o ingeniería financiera) cuya valoración del riesgo, en muchas ocasiones, es dudosa.

En las economías capitalistas Minsky identifica tres tipos de agentes: «entidades financieras cubiertas», «entidades especulativas» y «entidades Ponzi». Las primeras son aquellas que pueden responder a todas sus obligaciones de pago con los flujos de caja que generan. En las segundas, los flujos de caja generados no son suficientes para hacer frente al pago del principal de la deuda, por lo que vuelven a acudir al endeudamiento para no incurrir en impagos. Por último, las «entidades Ponzi» no tendrían recursos suficientes no solo para devolver el principal de sus deudas, sino tampoco para hacer frente al pago de los intereses.

Con esta estructura financiera de los agentes, cuando la burbuja explota (generalmente porque el precio de los activos ha llegado a niveles insostenibles o bien por cambios en el entramado regulatorio) la estructura de deudas de los agentes económicos ya no es coherente con los flujos financieros futuros y comienza un proceso de búsqueda de liquidez a través de la venta masiva de activos que lleva al colapso del valor de los mismos y al comienzo de la recesión. Es decir, cuando la burbuja explota, las entidades con falta de liquidez («entidades $\triangleright$

\footnotetext{
5 Minsky lo denomina «economías capitalistas».
} 
especulativas» y «entidades Ponzi») entran en bancarrota, incumpliendo sus obligaciones de pago y desplazando las «economías capitalistas» de una estructura financiera estable (dominadas por «entidades financieras cubiertas») a una estructura en crisis.

Este modelo de inestabilidad financiera también viene explicado por la existencia tanto de riesgo moral (que genera euforia en los inversores y una valoración incorrecta de los riesgos) como de fragilidad financiera (provocada principalmente por el surgimiento de instrumentos financieros innovadores). Este modelo explica en gran medida la crisis financiera internacional de 2009.

\section{Síntesis y conclusiones}

Las crisis financieras y monetarias que han acontecido desde el último cuarto del siglo $x x$ se han caracterizado por haberse presentado con formas diferentes y haber sido cambiantes. Es por ello que la teoría económica se ha ido adaptando a la realidad.

En este sentido, conviene destacar la importancia del estudio de las crisis de deuda. Estas crisis han afectado por igual a países en desarrollo (sobre todo de África) como a países emergentes (en América Latina, Europa del Este y Sudeste Asiático) y a países desarrollados (Estados Unidos o la zona euro durante la crisis financiera de 2009). Como se ha analizado, el modelo de Obstfeld y Rogoff (1995) demuestra que el endeudamiento externo es positivo para hacer un uso eficiente de los recursos. En este sentido, la propia Agenda de Acción de Addis Abeba (Naciones Unidas, 2015) reconoce el endeudamiento externo como un instrumento importante para financiar inversiones que permitan la consecución de los Objetivos de Desarrollo Sostenible, entre otros motivos porque los préstamos a Estados también permiten que las finanzas públicas desempeñen una función anticíclica en momentos de crisis. Ahora bien, hay que ser conscientes de que, si los recursos recibidos con dicha financiación no se utilizan para generar inversiones productivas, la dinámica de esta se volverá insostenible en el largo plazo, lastrando las posibilidades de desarrollo del país y generando justamente el efecto contrario al deseado (Argandoña et al., 1996). Por ello, es de suma importancia conocer la capacidad de un país para asumir un determinado nivel de endeudamiento, en función del crecimiento del PIB y del ritmo de crecimiento de su deuda ${ }^{6}$. En este sentido, el análisis teórico de las crisis financieras y monetarias complementa el estudio de la optimalidad del acceso al endeudamiento porque permite no solo conocer las ventajas de endeudarse con el exterior, sino también sus riegos.

Una de las conclusiones que se puede extraer del análisis teórico llevado a cabo es la importancia de que los países en desarrollo y emergentes diversifiquen sus fuentes de financiación al objeto de conseguir los recursos suficientes para el desarrollo económico y social. La inversión directa extranjera, el comercio internacional, la financiación combinada o «blended finance ${ }^{7}$ y las remesas se yerguen como instrumentos necesarios para conseguir este objetivo.

\footnotetext{
6 Pattillo, Poirson y Ricci (2002) estimaron que el crecimiento económico de un país se reduce un punto porcentual al duplicarse la deuda de dicho país. Reinhart y Rogoff (2009) estimaron que cuando el nivel de deuda externa de una economía emergente alcanza el 30-35 por 100 del producto nacional bruto, la probabilidad de que se produzca una crisis de deuda externa crece sustancialmente.

7 Supone mezclar los préstamos o inversiones en capital de entidades financieras o de desarrollo (públicas o privadas) con una subvención o donación (aportada generalmente por organismos internacionales).
} 


\section{Bibliografía}

[1] ALONSO, M. A.; BLANCO, F. J. (2004). «Los modelos de crisis gemelas en el marco de la literatura sobre crisis monetarias internacionales». Revista de Economía ICE, n.ำ 816.

[2] ARGANDOÑA, A.; GÁMEZ, C. y MOCHÓN, F. (1996). Macroeconomía avanzada I. Madrid: McGrawHill.

[3] BAJO, O. (2001). «Crisis Cambiarias: Teoría y Evidencia». Papeles de Trabajo del Instituto de Estudios Fiscales, n.․ 21/01.

[4] BARBERÁ, R. A. y BLANCA, M. (2004). «La interpretación de las crisis financieras a través de la literatura académica». Revista de Economía ICE, n.ㅇ 816 .

[5] FLOOD, R. y GARBER, P. (1984). «Collapsing exchange rate regimes: Some linear examples». Journal of International Economics, n.. 17, pp. 1-13.

[6] GANDOLFO, G. (2001). International finance and open-economy macroeconomics. Heidelberg, Springer.

[7] JACOBS, G. y RODRíGUEZ-ARANA, A. (2003). «Introducción. Crisis cambiarias y financieras: una comparación de dos crisis». Publicado como capítulo 1 del libro Crisis cambiarias y financieras. Una comparación de dos crisis. Madrid: Editorial Pirámide.

[8] JEANNE, O. y BENSAID, B. (1997). «The instability of fixed exchange rate systems when raising the nominal interest rate is costly". European Economic Review, Elsevier, vol. 41, n.. 8, pp. 1461-1478.

[9] KAMINSKY, G. y REINHART, C. (1999). «The Twin Crises: the causes of banking and balance-of-payments problems". The American Economic Review, vol. 89, n. 3, pp. 473-500.

[10] KINDLEBERGER, C.P. (1958). Economic development. Nueva York: McGraw Hill.
[11] KRUGMAN, P. (1999). «Balance Sheets, the Transfer Problem, and Financial Crises». International Tax and Public Finance, n. 6 , pp. 459-472.

[12] KYDLAND, F. y PRESCOTT, E. (1977). «Rules Rather than Discretion: The Inconsistency of Optimal Plans». Journal of Political Economy. June, pp. 473-492.

[13] MINSKY, H. (1993). «The financial instability hypothesis». Working paper, n.․ 74. The Jerome Levy Economics Institute of Bard College.

[14] NACIONES UNIDAS (2015). «Investing in Sustainable Development Goals. Action Plan for Private Investments in SDGs». UNCTAD/ OSG/2015/3 United Nations Publication. Genève 2015.

[15] NURKSE, R. (1995). Problemas de formación de capital en los países insuficientemente desarrollados. México, DF: Fondo de Cultura Económica.

[16] OBSTFELD, M. (1996). «Models of Currency Crises with Self-fulfilling Features". European Economic Review, vol. 40, n.ㅇ 3-5, pp. 1037-1047.

[17] OBSTFELD, M. y ROGOFF, K. (1995). Foundations of International Macroeconomics. Cambridge: The MIT Press.

[18] PATTILLO, C.; POIRSON, H. y RICCI, L. (2002). «External Debt and Growth». Nueva York: IFM Working Paper, 02/69.

[19] REINHART, C. y ROGOFF, K. (2009). «The aftermath of Financial Crises». American Economic Review, vol.98, n. 2, pp. 339-344.

[20] RODRIGUEZ PRADA, G. (2003). «La nueva economía de las crisis financieras en los mercados emergentes». Publicado como capítulo 2 del libro Crisis cambiarias y financieras. Una comparación de dos crisis. Madrid: Editorial Pirámide.

[21] ROSESTEIN-RODAN, P. (1961). Notes on the Theory of the Big Push. Ellis Editor. 


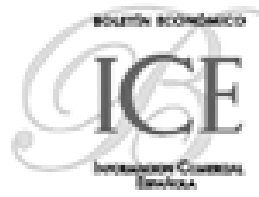

TÍTULOS PUBLICADOS EN 2017
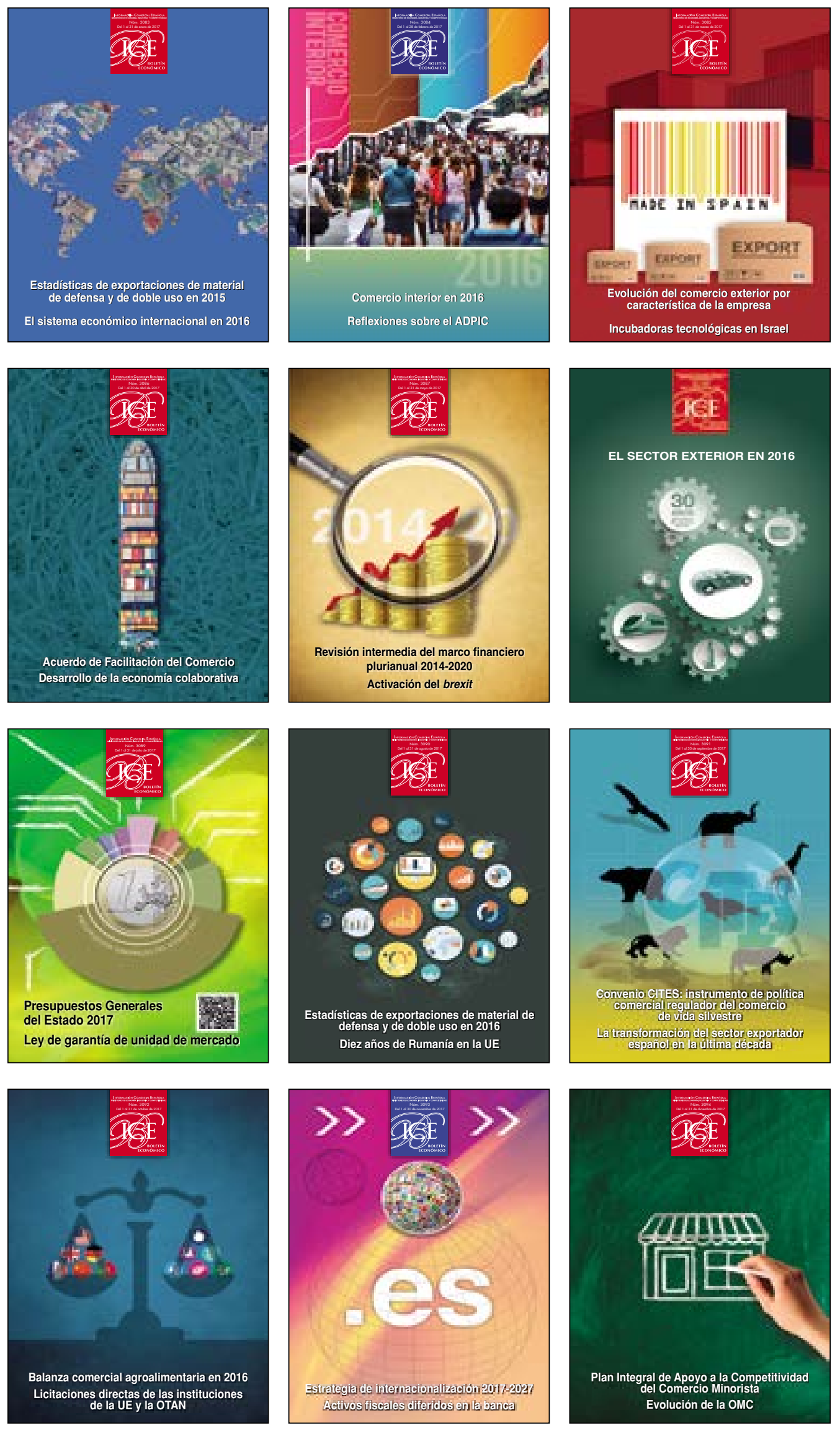

Página web: www.revistasice.com

Librería virtual, suscripción y venta: www.mineco.gob.es 